\title{
The Relationships Between Socioeconomic Status, COVID-19 Risk Perceptions, and the Adoption of Protective Measures in a Mid-Western City in the United States
}

\author{
Sarah Reed-Thryselius $s^{1,3} \cdot$ Lindsay Fuss ${ }^{2,3}\left[\right.$ Darren Rausch ${ }^{2}$ (D) \\ Accepted: 24 January 2022 / Published online: 7 February 2022 \\ (c) The Author(s), under exclusive licence to Springer Science+Business Media, LLC, part of Springer Nature 2022
}

\begin{abstract}
An individual's perception of risk plays an influential role in the behaviors they engage in, which could reduce or increase exposure or transmission of a certain disease. Since risk perceptions vary by social identities (e.g., gender, race/ethnicity, age) they are believed to influence the interpretation and likelihood of following guidance from risk-communication efforts. This study aims to understand how COVID-19 risk perceptions vary by social identity (with an emphasis upon socioeconomic factors), how such identities influence behavior adoption through risk-communication pathways, and how findings can be practically applied in messaging. Previous studies have investigated the role of social factors on risk perceptions, but SES has not been modeled as the main factor. Guided by the Health Belief Model and Social Determinant of Health Frameworks, findings from our 326 participants suggest those with high-risk COVID-19 perceptions identified as higher income and held more advanced educational degrees, suggesting a positive relationship between risk perceptions and SES. Individuals with high-risk perceptions more frequently reported practicing protective behaviors against COVID-19 and reported greater severity, susceptibility, barriers, benefits, trust, confidence, and health literacy in adopting behavior changes against the virus. When applying such findings to create a local risk-communication plan (logic model), it was found that messaging should be culturally relevant, in-plain language, and consistent to improve health literacy. In addition to using the most trusted and frequently used communication sources self-identified by residents, we recommend uniting trusted formal and informal community leaders to provide information in diverse pathways and formats.
\end{abstract}

Keywords Risk perceptions · Coronavirus (COVID-19) · Socioeconomic status (SES) · Health Belief Model (HBM) · Risk communication

Sarah Reed-Thryselius

Sarah.Reed@ho-chunk.com

Lindsay Fuss

Lindsay.Fuss@greenfieldwi.us

Darren Rausch

Darren.Rausch@greenfieldwi.us

1 Present Address: Ho-Chunk Nation Department of Health, N6250 Lumberjack Guy Road, Black River Falls, WI 54615, USA

2 Present Address: City of Greenfield Health Department, 7325 West Forest Home Avenue, Greenfield, WI 53220, USA

3 University of Wisconsin Milwaukee Joseph J. Zilber School of Public Health, 1240 12th Street, Milwaukee, WI 53205, USA

\section{Introduction}

The COVID-19 pandemic has reflected a disparity in morbidity and mortality outcomes [1], with the most impacted populations including lower socioeconomic status (SES) groups and racial/ethnic minorities [2]. This is problematic given that the pandemic may exacerbate existing social inequities [3]. Even in the city of Greenfield, Wisconsin, where $4.5 \%$ of residents identify as Hispanic/Latinx, this group accounts for $20-30 \%$ of labconfirmed COVID-19 cases since March 2020 [4]. With nearly 37,000 residents, like most Milwaukee County suburbs, the city experiences a resettlement gradient because of historical redlining practices [5]. In Greenfield, ACA 5 -year estimates show an east to west gradient, with neighborhoods situated on the most eastern side having a greater distribution of persons with lower SES and/or 
identifying as a racial/ethnic minority while the opposite is observed on the most western side.

In Social Determinant of Health (SDoH) frameworks, disease burden is often predicted by the historical context, the type of environmental conditions, and access to resources an individual or population experiences. Thus, groups with the greatest social disadvantage are more likely to experience the greatest burden of disease $[6,7]$. To our knowledge, previous COVID-19 risk perception studies have not modeled SES as the main variable of interest although it is theorized as a fundamental cause-highly preventable diseases often observe an SES disparity because resources can be used as buffers to prevent the occurrence or progression of the disease [8]. Because the Health Belief Model (HBM) conceptualizes risk perceptions as the result of modifying health factors [9], COVID-19 risk perceptions could be predicted by social factors, with SES as the dominant force, and this relationship could influence disease infection because of different access, exposure, and acceptance of risk communication messages [10].

HBM was first employed by the United States Public Health Service in the 1950s to understand why groups did not participate in preventative behaviors. These important constructs of the HBM include perceived susceptibility, perceived severity, perceived benefits, perceived barriers, cues to action, and self-efficacy; it has been applied to understand psycho-social and behavioral influences of disease on Human papillomavirus, colon cancer, tuberculosis [9] and used to guide risk communication models [11]. While risk communication can have a positive effect on managing emergencies, the polarized context of COVID-19 has posed many barriers to implement and achieve effective risk communication, including an everchanging climate of policy recommendations, scientific findings, and behavioral changes [12].

Patterns in risk perception are commonly observed in group identities. Higher risk perceptions were found among women, racial/ethnic minorities, immigrants, those with lower SES status [13, 14], and those with poor self-rated physical or mental health, living alone, or limited to no social support [15]. Political affiliation and level of trust in government were reported to be influential in several studies $[13,14]$ related to increased trust, knowledge, and behavior modification [16, 17]. Education did not have a direct effect on risk perceptions, but its effect was mediated through confidence in political leaders [14]. Some studies, however, found lower risk perceptions among lower SES and racial/ethnic groups because of their belief of limited autonomy to decrease their risk or exposure to COVID-19 due to material circumstances [18].

\section{Methods}

This study employed a stratified sampling process to investigate the populations in Greenfield, Wisconsin with the greatest differences in social advantage by sampling neighborhoods on the most eastern side and western side of the city. This social-geographical advantage assumption was verified through comparing neighborhood Social Vulnerability Index scores, Area Deprivation Index scores, and ACA 5-year estimates. Recruitment of participants began with a mailed welcome letter which included a link to access the survey. Four neighborhood tracts, two located in each region of the city, were randomly selected to receive such letters. Additional recruitment efforts occurred through social media platforms, but participants recruited through these channels were verified before including them in the sample. The inclusionary criteria required that participants must be 18 years or older, identify as a Greenfield resident, live within the defined sample region, and read/write in English as this was the only language materials were able to be produced in. The final sample included 326 respondents, resulting in a $15.2 \%$ response rate.

The employed risk perception survey was adapted from the WHO's Cosmo Protocol survey [19] but included one open-ended question which asked participants to describe how the pandemic affected them or their family's social, physical, mental, and economic wellbeing. This qualitative data was later coded for similar themes. Risk measurement items in the survey were scored and collapsed into seven categories, modeled off the HBM dimensions and previous studies employing a similar approach [16]. Response mean scores from each category ranged from 1 to 15 , with " 1 " indicating the lowest score and " 15 " indicating the highest score. One category assessed behavioral change, measured as adopting protective behaviors including mask wearing, social distancing, and avoiding large gatherings.

The main exposure variable was defined as SES, measured by educational attainment and household income, and the main outcome variable was defined as COVID19 risk perceptions, measured through the risk perception scoring scale. Although neighborhood location was collected in part of the sampling frame, it was not appropriate to include it in the regression tests because it breaks the assumption of independence [20]. All variables were defined as categorical and collapsed into related categories, determined from their distribution and the context associated with the category (Table 1). Risk perception was categorized into "high-risk" and "low-risk" determined by the median cut-point value. Education and household income were collapsed into "low, middle, and high" categories. Because household income was not associated with the risk level perception $(p=0.1778)$ but was 
Table 1 Distribution of demographic factors for "LowRisk" perceptions compared to "High-Risk" perception scorers$$
\text { Covar }
$$

Covariates

(N) Risk score

"Low-Risk"

Percent (\%)

Education
Low
Middle
High

Household income

Low

Middle

High

Race

Majority White

Majority non-White

Gender

Men

Women

Age

$18-34$

35-44

45-64

65-74

$75+$

Number of people in household

One

Two

Three or more

Work/school location

In-person

Hybrid/online

Neighborhood location

West

East

Physical health status

Excellent

Good

Fair/poor

Underlying health condition

Yes

No

Current smoker/vaper

Yes

No

Former smoker/vaper

Yes

No

HBM dimensions

Susceptibility

Severity

Self-efficacy

\section{2}

126

192

15.57

39.52

44.91

60

103

165

19.40

30.94

49.64

337

17

124

248

40

78

120

92

45

61

147

166

95.03

4.97

35.50

64.50

7.69

17.16

37.28

23.08

14.79

18.34

49.11

67.45

80

79

41.43

58.57

241

65.91

34.09

144

51

257

67

22.09

67.44

10.47

168

36.42

191

63.58

34

10.65

89.35

338

132

39.29

60.71

Mean

8.14 (2.74)

6.12 (4.04)

4.84 (1.89)
12.80

29.56

57.64

11.90

33.90

54.24

95.34

4.66

31.53

68.47

13.11

23.79

27.70

25.73

9.67

14.63

40.49

44.88

57.30

42.70

59.81

40.19

7.32

68.78

23.90

55.33

44.67

7.88

92.12

32.20

67.80

Score (SD)

$11.14(1.74)$

11.59 (2.60)

6.48 (2.52)
$<.0003^{* *}$

0.3573

$<.0001^{* *}$

$<.0001^{* *}$

$0.0491 * *$

0.1778

.8935

0.4183

$0.0491^{* *}$

$0.0508 *$

$0.0464 * *$

$<.0001 * *$ $<.0001 * *$ 
Table 1 (continued)

\begin{tabular}{|c|c|c|c|c|}
\hline \multirow[t]{3}{*}{ Covariates } & \multirow[t]{3}{*}{$(\mathrm{N})$} & \multicolumn{2}{|l|}{ Risk score } & \multirow[t]{3}{*}{$\mathrm{P}$ value } \\
\hline & & "Low-Risk" & "High-Risk" & \\
\hline & & \multicolumn{2}{|l|}{ Percent (\%) } & \\
\hline Barriers & & $1.25(1.86)$ & $2.81(2.90)$ & $<.0001 * *$ \\
\hline Benefits & & $5.34(1.95)$ & $7.00(1.78)$ & $<.0001^{* *}$ \\
\hline Cues to action & & $8.09(2.09)$ & 8.60 (1.97) & $0.0131 * *$ \\
\hline \multicolumn{5}{|l|}{ Behavior change } \\
\hline & & $12.14(3.51)$ & $13.47(1.87)$ & $<.0004 * *$ \\
\hline
\end{tabular}

Table 1 provides a summary of demographic factors and their value of association based on risk perception score

Education cut points are defined as "low" (high school degree or equivalent (GED) and having less than a high school degree), "medium" (associate degree and some college), or "high" (bachelor's degree or higher)

Household income accounts for household size and its cut points are defined as "low" $(\$ 35,000-\$ 49,000$ for two or more person households, $\$ 20,000-\$ 34,000$ for all person households, and less than $\$ 20,000$ for all person households), "medium" (\$75,000-\$99,000 for three to more person households, $\$ 50,000$ $\$ 74,999$ for all person households, and $\$ 35,000-\$ 49,000$ for one person household), or "high" ( $\$ 100,00$ or higher for all household sizes and $\$ 75,000-\$ 99,000$ for one to two person households)

SD Standard deviation

**Indicates significance at the 0.05 level. *Indicates significance at the 0.10 level

associated with education ( $\mathrm{p} \leq 0.0001)$, it was included in the final regression model, but education was modeled as the main exposure variable. While each HBM concept was assessed for its effect on COVID-19 risk perceptions, only the dichotomized risk score was used in the multivariate analysis because it is argued individual HBM concepts have minimal effects on a given outcome compared to its interaction among all concepts [9]. Based on its distribution, each concept's risk score was assessed for its association through t-tests and Wilcoxon-rank sum tests. Polychoric correlations were also run to assess the correlation of the frequency of engaging in a source/organization and its level of trust since trust and confidence are associated with risk perceptions [14, 16, 17]. A stepwise logistic regression followed these tests and additional association tests between the main outcome variable, main exposure variables, and other hypothesized confounders.

Following the completion of the study, an executive summary of the study's findings was provided to the health department to distribute to its residents to ensure transparency among the community and the department. Secondly, a risk-communication plan was developed from the findings, structured as a logic model (Fig. 1) based on the WHO's risk communication logic model [21]. The model provides the department with specific communication pathways, populations to mobilize, and/or resources to effectively tailor risk-communication messages. The logic model included the WHO's core components, such as areas of communication, including public information, mass mobilization, engagement, outputs, outcomes, and impact.

\section{Results}

Out of 326 eligible participants, those reporting a greater frequency of adopting COVID-19 protective behaviors also had significantly higher $(\mathrm{P} \leq 0.004)$ mean risk scores (13.41, SD 1.87) when compared to participants with lower risk scores (12.14, SD 3.51). Most participants identified as having a "high" educational attainment (40.2\%) or a "high" household income level (52.2\%). Moreover, of those participants who identified as "high" education, 63\% also identified as having a "high" income.

\section{COVID-19 Risk Perceptions}

As demonstrated in Table 1, characteristics of participants with significant high-risk perceptions included those identifying with "high" education, those aged 45-74 years, households with two or more people, those working/going to school in-person, those self-reporting their physical health status as "good," and those reporting an underlying health condition. Each HBM concept dimension produced statistically significant results. Those with high-risk perceptions reported greater barriers to be tested, greater benefits to avoid exposure, higher health literacy, and greater trust and confidence in information sources. In contrast, individuals with low-risk perceptions reported lower mean scores for barriers to be tested, benefits to avoid exposure, levels of susceptibility and severity, trust and confidence in information sources or health services, and health literacy. 
Fig. 1 The developed logic model for the City of Greenfield Health Department to support strategic COVID-19 risk communication

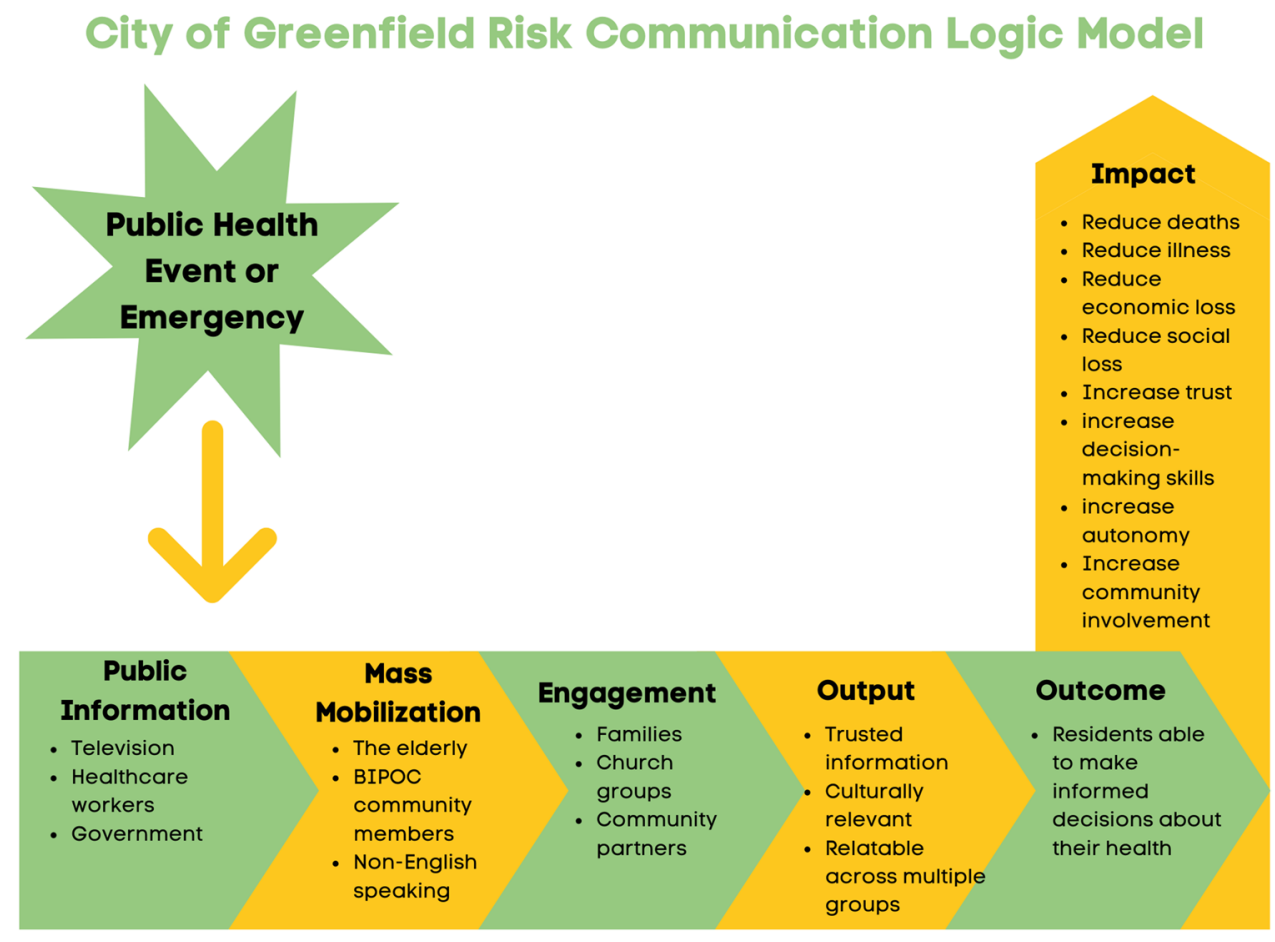

\section{Frequency, Trust, and Confidence}

Polychoric correlations suggested a strong, positive correlation between trust for a source and frequency of its use for all sources except healthcare workers as most of the sample already had high frequency $(82.8 \%)$ or high trust (40.6) for healthcare workers in comparison to the other sources. Most correlation values ranged from 0.7091 to 0.7835 but the value for healthcare workers was lower, around 0.4177 suggesting a moderate, positive relationship. Findings report that the sources of government, healthcare workers, and television were the most trusted and more likely to be used by a wide range of ages.

\section{Educational Attainment}

The significant associations of educational attainment included race/ethnicity, age, work/school location, selfrated physical health status, underlying health conditions, and current and former smoking status as described in Table 2. Individuals identifying as White (non-Hispanic), 35-64 years old, those working/attending school online or in a hybrid format, those with "excellent" or "good" self-rated health, those not having an underlying health condition, and non-current or former smokers/vapers had higher education levels when compared to their counterparts. The number of people in the household and gender were not statistically significant. Within education levels, "high" education had a greater percentage of participants who identified as having high-risk perceptions $(61.6 \%)$ in comparison to the percentages in "low" and "middle" education levels who identified as high-risk (50\% and $47.6 \%$ ). Of groups having high-risk perceptions, "high" education households made up $57.8 \%$ of all participants.

\section{Household Income}

The associations between household income and age, number of people in the household, work/school location, or physical health status, resulted in significant frequencies as reported in Table 3. Those aged 35-64 years, those with three or more persons in the household, working/going to school online or in a hybrid format, and those with "excellent" self-rated health had a greater percentage of participants who identified as high-income earners. Race/ethnicity, gender, and underlying health conditions were not statistically significant. Like education, high income earners were more than half of the participants reporting high-risk perceptions (54.2\%). Within income categories, "high" and "middle" income households had a greater percentage of individuals identifying as high-risk (58.1\% and 58.3\%) in comparison to "low" income households, who had a greater percentage of individuals with low-risk perceptions (43.8\%).

\section{SES Indicators}

Although a relationship between education and income is well documented, only educational attainment was significantly associated with risk perceptions $(\mathrm{p}=0.0491)$. While income was considered statistically insignificant 
Table 2 Distribution of demographic factors and bivariate associations for educational attainment categorized as "Low," "Middle," or "High"

\begin{tabular}{|c|c|c|c|c|c|}
\hline \multirow[t]{3}{*}{ Covariates } & \multirow[t]{3}{*}{$(\mathrm{N})$} & \multicolumn{3}{|c|}{ Educational level } & \multirow[t]{3}{*}{$P$ value } \\
\hline & & "Low" & "Middle" & "High" & \\
\hline & & & \multicolumn{2}{|l|}{ Percent $(\%)$} & \\
\hline \multicolumn{6}{|l|}{ Race } \\
\hline Majority White & 329 & 97.92 & 90.83 & 97.19 & $0.0279 * *$ \\
\hline Majority non-White & 17 & 2.08 & 9.17 & 2.81 & \\
\hline \multicolumn{6}{|l|}{ Gender } \\
\hline Men & 122 & 19.35 & 38.40 & 32.63 & 0.1304 \\
\hline Women & 245 & 80.65 & 61.60 & 67.37 & \\
\hline \multicolumn{6}{|l|}{ Age } \\
\hline $18-34$ & 39 & 11.54 & 7.94 & 12.00 & $0.0171 * *$ \\
\hline $35-44$ & 77 & 17.30 & 14.29 & 26.04 & \\
\hline $45-64$ & 117 & 21.16 & 38.10 & 30.21 & \\
\hline $65-74$ & 92 & 25.00 & 27.78 & 22.92 & \\
\hline $75+$ & 17 & 25.00 & 11.89 & 8.83 & \\
\hline Number of people in household & & & & & 0.3731 \\
\hline One & 61 & 23.08 & 13.60 & 16.75 & \\
\hline Two & 163 & 34.62 & 49.60 & 43.46 & \\
\hline Three or more & 144 & 42.30 & 36.80 & 39.79 & \\
\hline Work/school location & & & & & $0.0177 * *$ \\
\hline In-person & 78 & 57.14 & 64.81 & 40.70 & \\
\hline Hybrid/online & 76 & 42.89 & 35.19 & 59.30 & \\
\hline Neighborhood location & & & & & - \\
\hline West & 231 & 51.92 & 55.56 & 69.79 & \\
\hline East & 139 & 48.08 & 44.44 & 30.21 & \\
\hline Physical health status & & & & & $0.0136 * *$ \\
\hline Excellent & 50 & 8.06 & 8.73 & 18.89 & \\
\hline Good & 251 & 50.00 & 71.43 & 72.22 & \\
\hline Fair/poor & 67 & 41.94 & 19.83 & 8.89 & \\
\hline Underlying health condition & & & & & $0.0461 * *$ \\
\hline Yes & 167 & 51.02 & 55.37 & 41.21 & \\
\hline No & 185 & 48.98 & 44.63 & 58.79 & \\
\hline Current smoker/vaper & & & & & $0.0099 * *$ \\
\hline Yes & 33 & 15.69 & 12.80 & 4.76 & \\
\hline No & 332 & 84.31 & 87.20 & 95.24 & \\
\hline Former smoker/vaper & & & & & $<.0001 * *$ \\
\hline Yes & 130 & 51.92 & 47.20 & 23.28 & \\
\hline No & 236 & 48.08 & 52.80 & 76.72 & \\
\hline
\end{tabular}

Table 2 provides a summary of demographic factors and their value of association based on the educational attainment value

** Indicates significance at the 0.05 level

*Indicates significance at the 0.10 level $(\mathrm{p}=0.1778), 39 \%$ of all reported barriers were related to economic constraints. Yet, economics was also connected to themes of independence and government distrust within open-ended responses as one participant said, "It's ridiculous to me...that the government would basically kill businesses and their families because they think the best way to control the virus is isolate us and make mask mandates?" Another person shared similar sentiments, "Family businesses are closing permanently. These restrictions and mandates are killing more people than the virus. Enough! This must stop! Now!”.

\section{Multivariate Analysis}

As shown in Table 4, when comparing the odds of highrisk perceptions versus low-risk perceptions of "high" 
Table 3 Distribution of demographic factors and bivariate associations for income level households categorized as "Low," "Middle," or "High"

\begin{tabular}{|c|c|c|c|c|c|}
\hline \multirow[t]{3}{*}{ Covariates } & \multirow[t]{3}{*}{$(\mathrm{N})$} & \multicolumn{3}{|c|}{ Income level } & \multirow[t]{3}{*}{$\mathrm{P}$ value } \\
\hline & & "Low" & "Middle" & "High" & \\
\hline & & & Percent $(\%)$ & & \\
\hline \multicolumn{6}{|l|}{ Race } \\
\hline Majority White & 280 & 91.11 & 91.75 & 96.77 & 0.1516 \\
\hline Majority non-White & 17 & 8.89 & 8.25 & 3.23 & \\
\hline \multicolumn{6}{|l|}{ Gender } \\
\hline Men & 102 & 25.00 & 28.16 & 37.20 & 0.1518 \\
\hline Women & 213 & 75.00 & 71.84 & 62.80 & \\
\hline \multicolumn{6}{|l|}{ Age } \\
\hline $18-34$ & 40 & 14.58 & 12.62 & 12.12 & $0.0160 * *$ \\
\hline $35-44$ & 72 & 20.83 & 18.44 & 26.06 & \\
\hline $45-64$ & 100 & 22.93 & 26.21 & 37.58 & \\
\hline $65-74$ & 71 & 20.83 & 29.13 & 18.79 & \\
\hline $75+$ & 33 & 20.83 & 13.60 & 5.49 & \\
\hline Number of people in household & & & & & $<.0001 * *$ \\
\hline One & 50 & 29.17 & 28.16 & 4.24 & \\
\hline Two & 129 & 33.33 & 40.77 & 43.03 & \\
\hline Three or more & 137 & 37.50 & 31.07 & 52.73 & \\
\hline Work/school location & & & & & $0.0164 * *$ \\
\hline In-person & 72 & 80.00 & 57.14 & 42.35 & \\
\hline Hybrid/online & 70 & 20.00 & 42.86 & 57.65 & \\
\hline \multicolumn{6}{|l|}{ Neighborhood location } \\
\hline West & 187 & 52.08 & 54.37 & 64.24 & - \\
\hline East & 129 & 47.92 & 45.63 & 35.76 & \\
\hline Physical health status & & & & & $0.0080 * *$ \\
\hline Excellent & 43 & 8.33 & 9.71 & 17.56 & \\
\hline Good & 213 & 56.25 & 71.84 & 67.88 & \\
\hline Fair/poor & 60 & 35.42 & 18.45 & 14.56 & \\
\hline Underlying health condition & & & & & 0.5848 \\
\hline Yes & 146 & 48.89 & 51.49 & 45.00 & \\
\hline No & 160 & 51.11 & 48.51 & 55.00 & \\
\hline Current smoker/vaper & & & & & $0.0639 *$ \\
\hline Yes & 29 & 16.67 & 4.90 & 9.76 & \\
\hline No & 285 & 83.33 & 95.1 & 90.24 & \\
\hline Former smoker/vaper & & & & & $0.0540^{*}$ \\
\hline Yes & 113 & 50.00 & 36.89 & 31.10 & \\
\hline No & 202 & 50.00 & 63.11 & 68.90 & \\
\hline
\end{tabular}

Table 3 provides a summary of demographic factors and their value of association based on household income value

**Indicates significance at the 0.05 level

*Indicates significance at the 0.10 level educational groups compared to "middle" educational groups, this value was 0.323 (CI $0.120-0.873$ ). When the odds of high-risk perceptions compared to low-risk perceptions were investigated for "high" educational groups versus "low" educational groups, this value was 1.076 (CI $0.228-4.856$ ). While income was not associated with risk perceptions, because income was strongly associated with education, it was tested for interaction. The interaction term between income and education was significant for only lower income groups $(p=0.0277)$ and not for middle income groups $(p=0.7181)$. The final regression modeled risk perceptions as the outcome, educational attainment as the main exposure variable, an income interaction term, and other adjusted covariates of age, work location, underlying health conditions, self-rated health condition, and race/ethnicity. 
Table 4 Multivariate logistic regression for "High-Risk" versus "Low-Risk" perception score by educational attainment

\begin{tabular}{|c|c|c|c|}
\hline Education level & $\mathrm{N}$ & $\begin{array}{l}\text { OR of "High-Risk" versus } \\
\text { "Low-Risk" perception score }\end{array}$ & $95 \% \mathrm{CI}$ \\
\hline & 370 & Model 1 & \\
\hline Low & & 0.641 & $0.346,1.187$ \\
\hline Middle & & 0.583 & $0.370,0.918$ \\
\hline \multirow[t]{2}{*}{ High } & & Referent & \\
\hline & 137 & Model 2 & \\
\hline Low & & 0.924 & $0.240,3.555$ \\
\hline Middle & & 0.344 & $0.143,0.828$ \\
\hline \multirow[t]{2}{*}{ High } & & Referent & \\
\hline & 121 & Model 3 & \\
\hline Low & & 1.076 & $0.238,4.856$ \\
\hline Middle & & 0.323 & $0.120,0.873$ \\
\hline High & & Referent & \\
\hline
\end{tabular}

Model 1 = crude value

Model 2 adjusted for age, work/school location, self-rated physical health status, underlying health conditions, and race/ethnicity

Model 3 adjusted for age, work/school location, self-rated physical health status, underlying health conditions, and race/ethnicity; interaction term for income included

\section{Discussion}

Effective communication is vital during a health emergency to provide residents with essential information to protect themselves and others during an emergency. Since all HBM concept dimensions were significantly associated with risk perceptions, and individuals with higher risk engaged in protective behaviors more frequently and consistently compared to lower risk individuals, all HBM concepts should be applied in risk communication messages, supporting that such messages must identify with the specific subpopulations of a given community. Since residents are located within a spectrum of identifying their risk in relation to a health outcome, whether they identify with the recommendations given to avoid such health outcome, and whether they practice the suggested protective behaviors, communication should be targeted to a wide range of individuals while ensuring to identify with groups' risk perceptions, values, and beliefs.

Individuals with high-risk perceptions reported higher mean scores compared to individuals with low-risk perceptions across each HBM concept dimension suggesting that high-risk individuals felt more susceptible (likelihood to become infected) and reported more severe outcomes with the virus (e.g., social isolation, feeling helpless, or stressful). Although they had the greatest barriers to be tested (e.g., economic or stigma), they had greater benefits of avoiding exposure and protecting themselves. Individuals with highrisk perceptions also reported higher scores for self-efficacy, or the tools to make informed decisions about COVID-19 (measured as health literacy), and trust and confidence in sources or organizations who provide health education or health services. In contrast, those with low-risk perceptions reported lower scores for susceptibility, barriers to be tested, benefits to avoid exposure, health literacy, and trust and confidence in information sources or health services.

In our study, higher-risk scores among older individuals, larger households, those with an underlying health condition, or those in poorer physical health followed similar group risk perception patterns that other studies have observed [15]. However, we did not have statistically significant findings for gender or race/ethnicity that other studies have reported $[13,14]$. Social and mental health was an area that could likely influence COVID-19 risk perceptions as indicated by our text comments ( $61 \%$ of all comments coded for social or mental health) and were some of the most frequently selected perceived barriers to COVID-19 (e.g., social isolation, feeling helpless, or stressful). Some literature suggests that perceived stress [22] or more negative emotions, like feelings of anxiety, sadness, or loneliness may even mediate the effect of mental health outcomes on COVID-19 risk perceptions [23].

While we hypothesized that more socially disadvantaged groups would report higher risk perception scores, the opposite was observed in the fully adjusted logistic regression. From the reported OR and CI values, as shown in Table 4, it appears that when comparing the odds of participants with "high" education levels to participants with "middle" education levels for high-risk perceptions compared to low-risk perceptions, "middle" education has a protective effect on high-risk perceptions since its OR value is less than one (0.323, CI $0.120,0.873)$. When comparing the same odds, participants with "high" education compared to participants with "low" education levels, participants with "low" education have a slightly increased odds (1.076 times the odds) for high-risk perceptions but this OR is considered insignificant by CI standards. However, when assessing the interaction between education and income, the significant result among lower income households suggests the relationship between risk perceptions and SES, or the combinations between income and education groups and their relationship on risk perceptions, is more complex than previously hypothesized.

The contrasting ORs may be influenced by several factors including: (1) limited perceived individual autonomy that more socially disadvantaged individuals experience because they do not have the resources or opportunities to change their situation as Wolf, Serper, Opsanick et al. suggested [18], especially when the dominant message was to "stay home" although this may not be financially feasible or available for certain types of workers, (2) the increased trust and confidence in public health organizations that individuals with high-risk perception scores reported in comparison to 
individuals with lower-risk perceptions, (3) political affiliation, which is tied to income and education, but was not assessed because this study is facilitated in part with a government entity, and (4) a smaller sample size and a limited diverse sample, especially with more socially advantaged individuals since those who identified as "high" income households or with "high" educational attainment, had a greater percentage of respondents indicating high-risk perceptions when compared to their "middle" or "low" income or educational groups.

Because our study and previous studies support that risk perceptions influence behavior adoption [17, 24] and influence the effectiveness of risk communication [14, 16, 17], organizations responsible for risk communication should consider the effect of risk perceptions and how these perceptions vary by social identities. Moreover, because trust and confidence were common themes throughout our study and previous literature $[25,26]$, public health messaging efforts should be culturally relevant and consistent, clear, and inplain language [27]. Messaging should also be tailored to address the concerns or needs of different social groups, or this could contribute to mistrust, a perceived lack of empathy (they do not care about us), or ineffective communication.

Future studies should investigate the effect of social factors on risk perceptions because they are highly tied to the resources an individual/group can utilize as a buffer against conditions, activities, or social ties which may influence COVID-19 risk/exposure. Additionally, in future public health emergencies, particularly those which are perceived to be politically polarizing, it may be helpful to gather additional data on trust, confidence, and political affiliation as these factors may influence the effect of SES.

\section{Application}

The following section describes the application of our research findings, translating theory into practice for COVID-19 risk communication efforts through a logic model created for the Greenfield Health Department (Fig. 1). It provides public health professionals with a practical application of how research findings can support data-driven decisions, rooted in their community's contextual environment. The intention of the logic model is that residents can make informed decisions about their health through trustworthy, relatable, and culturally relevant communication messages.

From our study's findings, it was suggested that television, healthcare workers, and government were the most appropriate pathways since these sources were the most trusted and used by residents to receive information during the COVID-19 pandemic. When compared to other sources, a communication pathway not highly trusted nor frequently used for COVID-19 information was social media. While there was a wide age range for this study, it is important to identify that some residents may use certain sources over others because of access issues, lack of knowledge, unfamiliarity, or limited trust. Although social media sources were not rated as trustworthy as other sources, the health department tried to combat this by using a variety of sources such as Facebook or NextDoor. In theory, providing information in diverse formats may increase the likelihood that residents identify with at least one source. Regardless, it was recommended to establish trust within whichever selected pathway(s) before trying to communicate with residents.

Because language and its communication pathways must be culturally appropriate when providing widespread information about health emergencies to mobilize groups [28], it was recommended to mobilize the elderly, black, indigenous, and people of color (BIPOC), and non-English-speaking or English language learner residents. These three groups were chosen for several reasons: (1) many elderly residents identified as being particularly isolated during COVID-19 and unsure where to find COVID-19 information, especially since much of the health information was supported through social media and electronic-based platforms/devices, (2) the BIPOC populations were identified due to the recognition that communication needs to be culturally appropriate and be considerate of a wide range of community groups; in addition, some of these groups had high amounts of COVID19 cases despite small populations [4], and, (3) non-Englishspeaking and English language learners since the research survey was unable to be translated into additional languages and these members were excluded/limited from sharing their experiences.

For engagement, not only groups of individuals most likely to be impacted, but also families, informal and formal leaders, and other trusted sources within the community are important to effectively maintain population health and health equity work. For this reason, these groups were selected. Families were identified as a subgroup to identify with since Greenfield has several schools in its jurisdiction in addition to church groups and community partners. Because some religious/spiritual groups send members as part of a designated team to respond during emergencies and since the health department has an existing partnership with such groups, this connection could be a great resource to create and disseminate messaging as a trusted source for members. Other partnerships that are important to leverage include community sources that are integral to emergency response work such as fire/police departments, food banks, and healthcare services. In addition, groups with lower health literacy should be engaged. In the study, these groups also had lower risk perceptions, who also had less frequent behavior adoption. This may be due to a situation of conditions; these individuals may not have access to disposable income, the ability to work from home, or have health insurance which would limit 
their ability to take the necessary precautions and recommendations against COVID-19 (which was often, stay home or social distance). Many residents commented they were confused about where to go for testing and interpreting provided COVID-19 information as one resident said since, "guidelines were constantly changing.... and you really had to seek out the information on your own."

Lastly, in public health emergencies perceived as politically polarizing, politics should be considered as a factor to an individual/group's decision-making process; differing reports, political decisions, and "media hype" may create barriers to trust [16]. Residents left contrasting comments such as, "COVID has truly exposed how broken our government and healthcare system are in this country. To say our nation's response to a global pandemic was a failure would be an understatement. It is really unfortunate that science and public health have somehow become a political issue in our society and that there is a sizable percentage of our population (and also voters, no less) that deny science and don't follow simple rules encouraged by professionals," while another resident commented that, "The cure is worse than the infection for most people, be honest.... don't take away my freedom. Give facts and only facts and let each person decide." In these situations, in addition to using the most trusted and frequently used communication sources as self-identified by residents, we recommend uniting trusted formal and informal community leaders to provide consistent and transparent information to residents but in diverse pathways and formats. By creating similar messaging and sharing information widely it may increase participation in protective behaviors; it may also be beneficial since those who had greater awareness of the virus or had a higher perceived severity were more likely to tune in to media sourced by government officials [17].

\section{Limitations}

We recognize limitations in this study, including a lack of generalizability due to limited participation from a diverse range of community members. Due to funding delays and time constraints, the survey was not able to be translated into other languages and it was sent during the holiday, which may have influenced the response rate. Moreover, while our survey utilizes a validated risk perception survey, it focuses on variables that measure individual perspectives or actions; it does not account for the influence of others' behaviors/ actions which may increase communicable disease risk/ exposure in shared spaces or due to the demands or social expectations from social ties.
Authors Contributions SR-T and LF contributed to the study conception/design and material preparation. Epidemiological data analysis was performed by SR-T. Data collection, application of such findings, and creation of the logic model was performed by LF. The first draft of the manuscript was written by SR-T and LF. DR provided study support and manuscript assistance. All authors commented on previous versions of the manuscript and all authors read and approved the final manuscript.

Funding This study is supported by the Greenfield Health Department.

Availability of Data and Material The datasets generated during and/ or analyzed during the current study are not publicly available due to IRB agreements but the adapted COVID-19 Risk Perception Scale used during this study is available from the corresponding author on reasonable request.

Code Availability The code generated during the study's analysis is not available due to program availability limitations after leaving the institution at which the study was completed.

\section{Declarations}

Conflict of interest All authors were employed at the Greenfield Health Department during the study, but the study is within normal public health programming and COVID-19 response. The authors report no other competing interests.

Consent to Participate All participants agreed to participate through an informed consent process, which was required as part of the IRB's (mentioned above) ethical guidelines.

Consent for Publication Lindsay Fuss holds full ownership of the logic model presented in this study and consents to its publishing in this scope.

Ethics Approval This study meets all ethical guidelines and was approved by the UW-Milwaukee Institutional Review Committee (IRB), per study \# 21.135.

\section{References}

1. Douglas, J. A., \& Subica, A. M. (2020). COVID-19 treatment resource disparities and social disadvantage in New York City. Preventive Medicine, 141, 106282. https://doi.org/10.1016/j. ypmed.2020.106282

2. Rozenfeld, Y., Beam, J., Maier, H., Haggerson, W., Boudreau, K., Carlson, J., \& Medows, R. (2020). A model of disparities: Risk factors associated with COVID-19 infection. International Journal for Equity in Health, 19(126), 2-10.

3. Bambra, C., Riordan, R., Ford, J., \& Matthews, F. (2020). The COVID-19 pandemic and health inequalities. Journal of Epidemiology and Community Health. https://doi.org/10.1136/ jech-2020-214401

4. Greenfield Health Department. (2021, October 12). Weekly snapshot for City of Greenfield, week ending on October 9, 2021. City of Greenfield Wisconsin. Retrieved October 17, 2021, from https://www.ci.greenfield.wi.us/1250/Weekly-Snapshot-City-ofGreenfield

5. Foltman, L., Jones, M., \& Bourbeau, C. (2019, February 28). How redlining continues to shape racial segregation in Milwaukee. UW Applied Population Lab. Retrieved January 23, 2022, from https:// 
www.wiscontext.org/how-redlining-continues-shape-racial-segre gation-milwaukee

6. Birn, A-E., Pillay, Y., \& Holtz, T. H. (2017). Political economy of health and development. In Textbook of global health. pp. 101-104. Oxford University Press.

7. Solar, O., \& Irwin, A. (2010). A conceptual framework for action on the social determinants of health. Social Determinants of Health Discussion Paper 2 (Policy and Practice) (pp. 3-75). World Health Organization.

8. Phelan, J. C., Link, B. G., \& Tehranifar, P. (2010). Social conditions as fundamental causes of health inequalities: Theory, evidence, and policy implications. Journal of Health and Social Behavior, 51(S), S28-S40.

9. Glanz, K., Rimer, B. K., \& Viswanath, K. (2015). Health behavior: Theory, research, and practice. Jossey-Bass.

10. Usuwa, I. S., Akpa, C. O., Umeokonkwo, C. D., Umoke, M., Oguanuo, C. S., Olorukooba, A. A., Bamgboye, E., \& Balogun, M. S. (2020). Knowledge and risk perception towards Lassa fever infection among residents of affected communities in Ebonyi State, Nigeria: Implications for risk communication. BMC Public Health, 20(1), 1-10. https://doi.org/10.1186/s12889-020-8299-3

11. World Health Organization [WHO]. (2020, January 15). Emergencies: Risk communication. World Health Organization. Retrieved December 29, 2021, from https://www.who.int/news-room/quest ions-and-answers/item/emergencies-risk-communication\#: : text=Risk\%20communication\%20refers\%20to\%20the,economic\% 20or\%20social\%20well\%2Dbeing

12. Paulik, L. B., Keenan, R. E., \& Durda, J. L. (2020). The case for effective risk communication: Lessons from a global pandemic. Integrated Environmental Assessment and Management, 16(5), 552-554. https://doi.org/10.1002/ieam.4312

13. Nivette, A., Ribeaud, D., Murray, A., Steinhoff, A., Bechtiger, L., Hepp, U., Shanahan, L., \& Eisner, M. (2021). Non-compliance with COVID-19-related public health measures among young adults in Switzerland: Insights from a longitudinal cohort study. Social Science \& Medicine, 268(113370), 1-9.

14. Shao, W., \& Hoa, F. (2020). Confidence in political leaders can slant risk perceptions of COVID-19 in a highly polarized environment. Social Science \& Medicine, 261(11325), 1-6.

15. Laires, P. A., Dias, S., Gama, A., Moniz, M., Pedro, A. R., Soares, P., Aguiar, P., \& Nunes, C. (2021). The association between chronic disease and serious COVID-19 outcomes and its influence of risk perception: Survey study and database analysis. JMIR Public Health Surveillance, 7(1), e22794.

16. Jose, R., Narendran, M., Bindu, A., Beevi, N., Manju, L., \& Benny, P. V. (2021). Public perception and preparedness for the pandemic COVID 19: A health belief model approach. Clinical Epidemiology Global Health, 9, 41-46. https://doi.org/10.1016/j. cegh.2020.06.009

17. Ning, L., Niu, J., Bi, X., Yang, C., Liu, Z., Wu, Q., Ning, N., Liang, L., Liu, A., Hao, Y., Gao, L., \& Liu, C. (2020). The impacts of knowledge, risk perception, emotion and information on citizens' protective behaviors during the outbreak of COVID-19: A cross-sectional study in China. BMC Public Health. https://doi. org/10.1186/s12889-020-09892-Y

18. Wolf, M. S., Serper, M., Opsasnick, L., O'Conor, R. M., Curtis, L. M., Benavente, J. Y., Wismer, G., Batio, S., Eifler, M., Zheng,
P., Russell, A., Arvanitis, M., Ladner, D., Kwasny, M., Persell, S. D., Rowe, T., Linder, J. A., \& Bailey, S. C. (2020). Awareness, attitudes, and actions related to COVID-19 among adults with chronic conditions at the onset of the U.S. Outbreak. Annals of Internal Medicine. https://doi.org/10.7326/M20-1239

19. World Health Organization [WHO]. WHO Cosmo Protocol Survey. WHO; 2019. COVID-19 survey tool and guidance. Retrieved October 17, 2021, from https://www.euro.who.int/_data/assets/ pdf_file/0007/436705/COVID-19-survey-tool-and-guidance.pdf

20. Chakraborty, J., Maantay, J. A., \& Brender, J. D. (2011). Disproportionate proximity to environmental health hazards: Methods, models, and measurement. American Journal of Public Health, 101(S1), S27-S36.

21. World Health Organization [WHO]. N.d. Risk communication strategies module B3. World Health Organization. Retrieved December 29, 2021, from https://www.who.int/risk-communicat ion/training/Module-B3.pdf

22. Li, X., \& Lyu, H. (2021). Epidemic risk perception, perceived stress, and mental health during COVID-19 pandemic: A moderated mediating model. Frontiers Psychology. https://doi.org/10. 3389/fpsyg.2020.563741/full

23. Han, Q., Zheng, B., Agostini, M., Bélanger, J. J., Gützkow, B., Kreienkamp, J., Reitsema, A. M., \& van Breen, J. A. (2021). PsyCorona collaboration, \& pontus leander, N. Journal of Affective Disorder, 284, 246-255.

24. Seehuus, M., Stanton, A. M., Handy, A. B., Haik, A. K., Gorman, R., \& Clifton, J. (2021). Impact of COVID-19 predicts perceived risk more strongly than known demographic risk factors. Journal Psychosomatic Research, 140, 110299.

25. Lohiniva, A., Sane, J., Sibenberg, K., Puumalainen, T., \& Salminen, M. (2020). Understanding coronavirus disease (COVID-19) risk perceptions among the public to enhance risk communication efforts: A practical approach for outbreaks, Finland, February 2020. Eurosurveillance. https://doi.org/10.2807/1560-7917. es.2020.25.13.2000317

26. Zhang, L., Li, H., \& Chen, K. (2020). Effective risk communication for public health emergency: Reflection on the COVID-19 (2019-nCoV) outbreak in Wuhan, China. Healthcare, 8(1), 64. https://doi.org/10.3390/healthcare8010064

27. Chess, C., Burger, J., \& McDermott, M. H. (2005). Speaking like a state: Environmental justice and fish consumption advisories. Society \& Natural Resources, 18(3), 267-278. https://doi.org/10. 1080/08941920590908132

28. Wray, R. J., Becker, S. M., Henderson, N., Glik, D., Jupka, K., Middleton, S., Henderson, C., Drury, A., \& Mitchell, E. W. (2008). Communicating with the public about emerging health threats: Lessons from the pre-event message development project. American Journal of Public Health, 98(12), 2214-2222. https:// doi.org/10.2105/ajph.2006.107102

Publisher's Note Springer Nature remains neutral with regard to jurisdictional claims in published maps and institutional affiliations. 